\title{
Métodos transientes de troca de calor na determinação das propriedades térmicas de materiais cerâmicos: II - o método do fio quente
}

\author{
(Thermal properties of ceramic materials by the non-steady techniques: \\ II - the hot wire technique)
}

\author{
W. N. dos Santos \\ Departamento de Engenharia de Materiais, Universidade Federal de S. Carlos, Rodovia Washington Luiz, km 235, \\ CP 676, S. Carlos, SP 13565-905 \\ dwns@power.ufscar.br
}

\begin{abstract}
Resumo
A condutividade térmica, a difusividade térmica e o calor específico, conhecidos como propriedades térmicas são as três propriedades físicas de um material mais importantes do ponto de vista de cálculos térmicos. Elas são essenciais na determinação dos parâmetros de resistência ao choque térmico em refratários e na determinação dos fatores de dissipação de calor em fornos e fornalhas. A condutividade térmica é um parâmetro importante em problemas envolvendo transferência de calor em regime estacionário, enquanto que a difusividade térmica é importante em estados não estacionários de troca de calor. O calor específico, propriedade importante em altas temperaturas, está associado ao consumo de energia durante o aquecimento. Normalmente essas três propriedades são determinadas separadamente por técnicas e equipamentos individuais. Hoje são conhecidos vários métodos para a determinação da condutividade térmica e difusividade térmica. Recentemente, os métodos transientes de troca de calor têm sido os métodos preferidos na determinação das propriedades térmicas de materiais. Neste trabalho é feita uma descrição e uma análise crítica de um dos métodos mais utilizados na determinação das propriedades térmicas de materiais cerâmicos: o método do fio quente. Com este método é possível determinar simultaneamente, a partir do mesmo transiente térmico experimental, as três propriedades térmicas. Resultados numéricos são também apresentados.

Palavras-chave: métodos transientes, propriedades térmicas, condutividade térmica, calor específico, difusividade térmica, método do fio quente.
\end{abstract}

\begin{abstract}
Thermal conductivity, thermal diffusivity and specific heat, namely as the thermal properties, are the three most important physical properties of a material that are needed for heat transfer calculations. They are essential in thermal shock parameters determination or in calculations of heat dissipation factors in furnaces and kilns. Thermal conductivity is an important parameter in problems involving steady state heat transfer, while the thermal diffusivity is important in non-steady heat transfer calculations. The specific heat is also a decisive property of ceramic materials in high temperature applications, and it is associated with heat consumption during the heating process. Normally these properties are determined separately by individual techniques and apparatus. Nowadays, several different techniques for the determination of the thermal diffusivity and thermal conductivity may be found in the literature. Recently, transient techniques have become the preferable way for measuring thermal properties of materials. In this work it is presented a description as well as a critical analysis of one experimental technique employed worldwide for thermal properties measurements of ceramic materials: the hot wire technique. With this technique it is possible to determine simultaneously from the same temperature transient the three thermal properties. Numerical results are also presented.
\end{abstract}

Keywords: transient techniques, thermal properties, thermal conductivity, thermal diffusivity, specific heat, hot wire technique.

\section{INTRODUÇÃO}

A condutividade térmica, a difusividade térmica e o calor específico, conhecidas como propriedades térmicas ou termofísicas são as três propriedades físicas de um material mais importantes do ponto de vista de cálculos térmicos. Elas são essenciais na determinação dos parâmetros de resistência ao choque térmico em refratários e na determinação dos fatores de dissipação de calor em fornos e fornalhas. A condutividade térmica é a propriedade que estabelece os níveis de temperatura de trabalho de um material, sendo um parâmetro importante em problemas envolvendo transferência de calor em regime estacionário. A difusividade térmica é uma medida da rapidez com a qual o calor se propaga através do material e é importante em problemas envolvendo estado não estacionário de trocas de calor. O calor específico é uma quantidade termodinâmica, importante em altas temperaturas, e está associado ao consumo de energia durante o aquecimento. Normalmente essas três propriedades são determinadas separadamente por 
técnicas e equipamentos individuais. Elas estão relacionadas entre si pela equação:

$$
\mathrm{a}=\frac{\mathrm{k}}{\rho \mathrm{c}_{\mathrm{p}}}
$$

onde: $\mathrm{a}=$ difusividade térmica $\left(\mathrm{m}^{2} / \mathrm{s}\right), \mathrm{k}=$ condutividade térmica $(\mathrm{W} / \mathrm{mK}), \rho=$ densidade $\left(\mathrm{kg} / \mathrm{m}^{3}\right)$ e $\mathrm{c}_{\mathrm{p}}=$ calor específico a pressão constante $(\mathrm{J} / \mathrm{kgK})$.

Hoje são conhecidos vários métodos para a determinação da condutividade térmica e difusividade térmica de um material. Recentemente, os métodos transientes de troca de calor têm sido os métodos preferidos na determinação das propriedades térmicas de materiais. Neste trabalho é feita uma descrição e uma análise crítica de um dos métodos mais utilizados na determinação das propriedades térmicas de materiais cerâmicos: o método do fio quente. Com este método é possível determinar simultaneamente, a partir do mesmo transiente térmico experimental, as três propriedades térmicas.

O método do fio quente, descrito por Schieirmacher [1] in 1888 , é um método absoluto, direto e não estacionário na determinação da condutividade térmica de um material. Van Der Held e Van Drunen [2] em 1949 foram os que pela primeira vez fizeram uso prático dessa técnica de medida. Entretanto, foi Haupin [3] em 1960 que utilizou pela primeira vez o método do fio quente na determinação da condutividade térmica de materiais cerâmicos, e seu trabalho formou a base de todas as variantes do método.

Hoje em dia o método do fio quente é considerado como uma técnica precisa na determinação da condutividade térmica de materiais cerâmicos. Nesta técnica de medida, é eliminado o conceito de "temperatura média" entre a face quente e a face fria de uma amostra utilizado nos cálculos dos métodos calorimétricos, uma vez que o cálculo da condutividade é feito numa determinada temperatura fixa. Nesta técnica, o gradiente de temperatura através da amostra é muito baixo, o que é sem dúvida outra virtude deste método uma vez que um método ideal de medida de condutividade térmica seria aquele capaz de medir essa propriedade segundo um gradiente de temperatura zero através da amostra.

Entretanto, este método tem duas limitações: materiais condutores elétricos, e materiais de alta condutividade térmica. No primeiro caso a solução seria obtida isolandose eletricamente o fio quente e as amostras. Já para o caso de materiais de alta condutividade térmica, o tempo de registro do transiente térmico torna-se bastante pequeno, comprometendo assim a confiabilidade dos resultados obtidos, a menos que as dimensões das amostras sejam suficientemente grandes, o que por outro lado inviabiliza o processo de medida.

\section{FUNDAMENTOS TEÓRICOS}

Se um material é isotrópico, a condutividade térmica é a mesma em qualquer direção.
A temperatura $\mathrm{T}$, no instante $\mathrm{t}$ e no ponto $(\mathrm{x}, \mathrm{y}, \mathrm{z})$, num sólido infinito, devido a uma quantidade de calor q que é instantaneamente gerada no tempo $\mathrm{t}=0$ no ponto $\left(\mathrm{x}^{\prime}, \mathrm{y}^{\prime}, \mathrm{z}^{\prime}\right)$, é dada pela equação [4]:

$\mathrm{T}=\frac{\mathrm{q}}{8 \rho \mathrm{c}(\pi \mathrm{at})^{3 / 2}} \exp \left\{-\frac{\left(\mathrm{x}-\mathrm{x}^{\prime}\right)^{2}+\left(\mathrm{y}-\mathrm{y}^{\prime}\right)^{2}+\left(\mathrm{z}-\mathrm{z}^{\prime}\right)^{2}}{4 \mathrm{at}}\right\}(\mathrm{B})$

onde: $\rho=$ densidade, $\mathrm{c}=$ calor específico, $\mathrm{a}=\mathrm{k} / \rho \mathrm{c}=$ difusividade térmica.

Para uma fonte linear instantânea de calor, se uma quantidade de calor q'dz é instantaneamente gerada no instante $\mathrm{t}=0$, em todos os pontos sobre uma linha infinita paralela ao eixo z, e passando pelo ponto (x', y'), a temperatura no ponto $(\mathrm{x}, \mathrm{y})$ no instante $\mathrm{t}$ é obtida substituindo-se q na equação B por q'dz, e integrando com respeito a $z$ :

$\mathrm{T}=\frac{\mathrm{q}^{\prime}}{4 \pi \mathrm{kt}} \exp \left\{-\frac{\left(\mathrm{x}-\mathrm{x}^{\prime}\right)^{2}+\left(\mathrm{y}-\mathrm{y}^{\prime}\right)^{2}}{4 \mathrm{at}}\right\}$

sendo q' a quantidade de calor gerada por unidade de comprimento da fonte.

Se uma fonte gera calor a partir do instante $\mathrm{t}=0$, a uma taxa $q^{\prime}(t)$ por unidade de tempo e por unidade de comprimento de uma linha paralela ao eixo $\mathrm{z}$ passando pelo ponto ( $\mathrm{x}$, $\left.y^{\prime}\right)$, a temperatura no ponto $(x, y)$ no instante $t$ é obtida substituindo-se q' na equação $C$ por q'( $(t) d t$ e integrando com respeito a t:

$\mathrm{T}=\frac{\mathrm{q}^{\prime}}{4 \pi \mathrm{k}} \int_{\mathrm{r}^{2} / 4 \mathrm{at}}^{\infty} \frac{\mathrm{e}^{-\mathrm{u}}}{\mathrm{u}} \mathrm{du}=\frac{\mathrm{q}^{\prime}}{4 \pi \mathrm{k}}\left[-\operatorname{Ei}\left(-\frac{\mathrm{r}^{2}}{4 \mathrm{at}}\right)\right]$

onde $\mathrm{r}^{2}=\left(\mathrm{x}-\mathrm{x}^{\prime}\right)^{2}+\left(\mathrm{y}-\mathrm{y}^{\prime}\right)^{2}$, e q'é a quantidade de calor gerada por unidade de comprimento da fonte.

A função - Ei(-x), chamada função exponencial integral é definida por:

$$
-\operatorname{Ei}(-\mathrm{x})=\int_{\mathrm{x}}^{\infty} \frac{\mathrm{e}^{-\mathrm{t}}}{\mathrm{t}} \mathrm{dt}
$$

e pode ser aproximada pela seguinte série de potências:

$-\operatorname{Ei}(-x)=\operatorname{E} 1(x)=-\gamma-\ln x-\sum_{n=1}^{\infty} \frac{(-1)^{n} x^{n}}{n n !}$

desde que $|\arg \mathrm{x}|<\pi$, e $\arg \mathrm{x}=\arctan \left(\mathrm{x}_{2} / \mathrm{x}_{1}\right)$, sendo $\mathrm{x}=$ $\mathrm{x}_{1}+\mathrm{i} \mathrm{x}_{2}, \mathrm{e} \gamma=0,5772156649 \ldots$ é a constante de Euler.

Para uma formulação matemática do método, o fio quente é assumido ser uma fonte de calor ideal (massa $=0$, 
$\mathrm{e}$, portanto, capacidade térmica $=0$ ), infinitamente longa e fina (diâmetro $=0$ ), a qual é circundada até o infinito pelo material cuja condutividade térmica pretende-se determinar [5]. Esta hipótese implica que o transiente de temperatura que é registrado pelo termopar no ponto de medida durante o experimento não pode ser alterado pelo fato da amostra real ter dimensões finitas.

Ao passar uma corrente elétrica constante através do fio, uma quantidade constante de calor, por unidade de tempo e por unidade de comprimento, é liberada pelo fio e vai se propagar através do material. Essa propagação de calor num meio infinito gera, no material, um campo transiente de temperaturas.

Na prática, a fonte teórica linear é aproximada por uma resistência elétrica fina, e o sólido infinito é substituído por uma amostra finita. Assim sendo, a capacidade térmica do fio, a resistência de contato entre ele e a amostra, e o tamanho finito da amostra são fatores que impõem um tempo mínimo e um tempo máximo a serem utilizados no cálculo da condutividade térmica.

A diferença entre as curvas temperatura versus tempo, teórica e real, quando se utiliza dois (paralelo e superfície) dos quatro possíveis arranjos experimentais desse método é mostrada na Fig. 1.

A diferença entre as curvas real e teórica em seu trecho

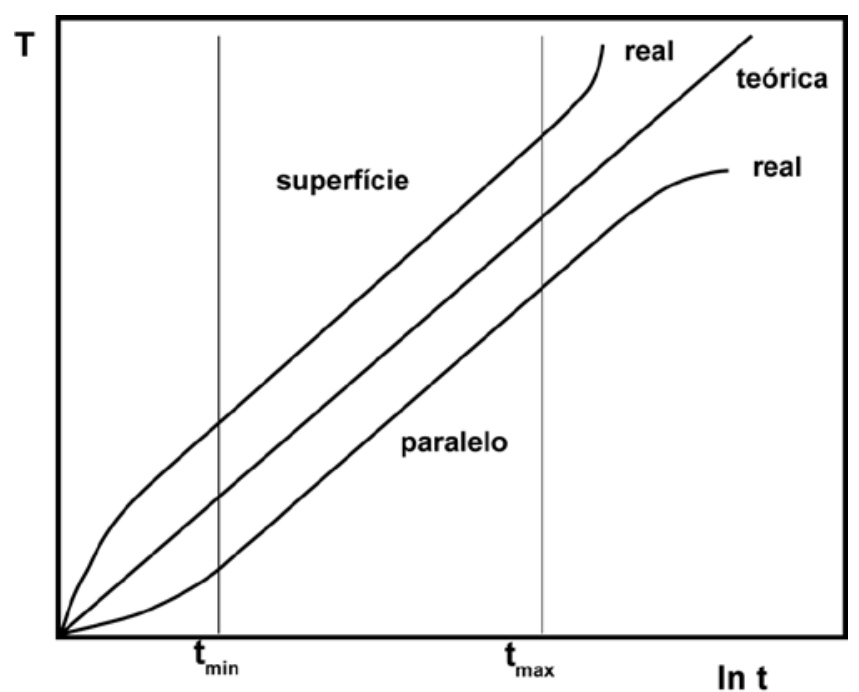

Figura 1: Curvas temperatura versus tempo.

[Figure 1: Actual and theoretical time-temperature curves.]

inicial é devido à resistência de contato entre o fio quente e a amostra e à inércia térmica do material, enquanto que a diferença no trecho final é conseqüência do tamanho finito da amostra. A região intermediária, onde as curvas real e teórica exibem o mesmo comportamento, define os limites de tempo $\left(\mathrm{t}_{\text {min }}\right.$ e $\left.\mathrm{t}_{\text {max }}\right)$ que devem ser considerados no cálculo da condutividade térmica pela técnica de fio quente. Assim, o tempo máximo de medida decresce com o aumento da condutividade térmica do material a ser ensaiado. Esse fato implica em uma outra limitação à aplicação deste método para materiais metálicos, que tendo alta condutividade térmica reduziriam drasticamente o tempo máximo de medida.

Alguns cuidados devem ser tomados ao se trabalhar com o método do fio quente para assegurar resultados precisos e consistentes [6]: 1. utilizar uma resistência elétrica fina, que se aproxime o máximo possível da fonte de calor linear teórica; 2. assegurar o melhor contato possível entre ambas as amostras e o fio quente, reduzindo assim os efeitos da resistência de contato; 3. não considerar, para efeito de cálculo, o trecho inicial da curva, eliminando assim os efeitos de contato térmico entre a resistência elétrica e o material da amostra; 4. limitar o tempo de ensaio para assegurar que o tamanho finito da amostra não afete as temperaturas medidas.

\section{VARIANTES DO MÉTODO DE FIO QUENTE}

Arranjo de Haupin - No arranjo inicial, descrito por Haupin, na primeira utilização do método de fio quente [7] na determinação da condutividade térmica de materiais cerâmicos, um termopar é utilizado como fio quente e sensor de temperatura ao mesmo tempo, tendo uma de suas junções colocadas no centro da amostra. A outra junção do termopar é colocada numa garrafa térmica contendo gelo fundente, que é utilizada como fonte de referência de temperatura. $\mathrm{O}$ fio é aquecido por corrente alternada, e um filtro é utilizado para bloquear a corrente alternada no circuito de medida da temperatura, permitindo, assim, medir a força eletromotriz gerada pelo termopar com o auxílio de um voltímetro. A Fig. 2 ilustra esse arranjo experimental.

Esse arranjo requer uma fonte de potência constante com uma saída senoidal muito boa para que a filtragem seja eficiente. Além disso, é necessária uma blindagem eficiente para o filtro com o objetivo de impedir interferência eletrônica. Uma vez que o termopar, também usado como fio quente é constituído por dois metais diferentes com diferentes propriedades térmicas e elétricas, o aquecimento nunca é absolutamente constante de uma extremidade a outra do fio. Haupin utilizou um fator de correção para compensar as flutuações térmicas causadas por esse problema. Esse arranjo

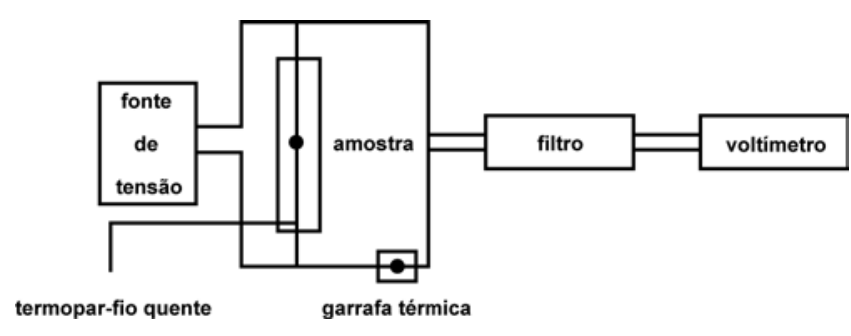

Figura 2: Arranjo experimental de Haupin.

[Figure 2: Haupin experimental set-up.] 
não é utilizado atualmente, e sua importância é histórica por ter sido o primeiro arranjo experimental na determinação da condutividade térmica de materiais cerâmicos.

Técnica padrão de fio quente (cruz de medição) - Essa técnica foi desenvolvida por Mittenbuhler [8]. É aplicada a materiais com densidade superior a $500 \mathrm{~kg} / \mathrm{m}^{3}$, sendo adequada para materiais cuja condutividade térmica seja inferior a 2,0 W/mK, e cuja difusividade térmica seja inferior a $8,333 \times 10^{-7} \mathrm{~m}^{2} / \mathrm{s}$. Nessa técnica, uma junção do termopar é soldada no meio do fio quente. A variação da temperatura no fio, que é função da condutividade térmica do material, é medida em função do tempo. Para a colocação da resistência elétrica e do termopar, são usinadas em uma das faces de uma das amostras duas ranhuras ortogonais para receber a cruz de medição e uma ranhura em $\mathrm{V}$ para receber a junção de referência do termopar. A profundidade desses sulcos deve corresponder aproximadamente ao diâmetro dos fios a serem aí inseridos. A cruz de medição é formada pelo fio quente e pelo termopar, que tem uma de suas junções soldada perpendicularmente ao fio. A junção de referência do termopar é colocada a cerca de $10 \mathrm{~mm}$ da extremidade do corpo de prova. Após a colocação desses fios, os sulcos são preenchidos com uma massa feita com pó do mesmo material misturado com $2 \%$ de dextrina. Sobre esse corpo de prova é então colocado o segundo corpo. As superfícies em contato devem ser bem polidas, para proporcionar o melhor contato térmico possível. A Fig. 3 mostra um diagrama esquemático da técnica descrita.

Essa técnica foi normalizada em 1976 para norma DIN 51046-Parte 1 [9]. A condutividade térmica é calculada a partir da equação:

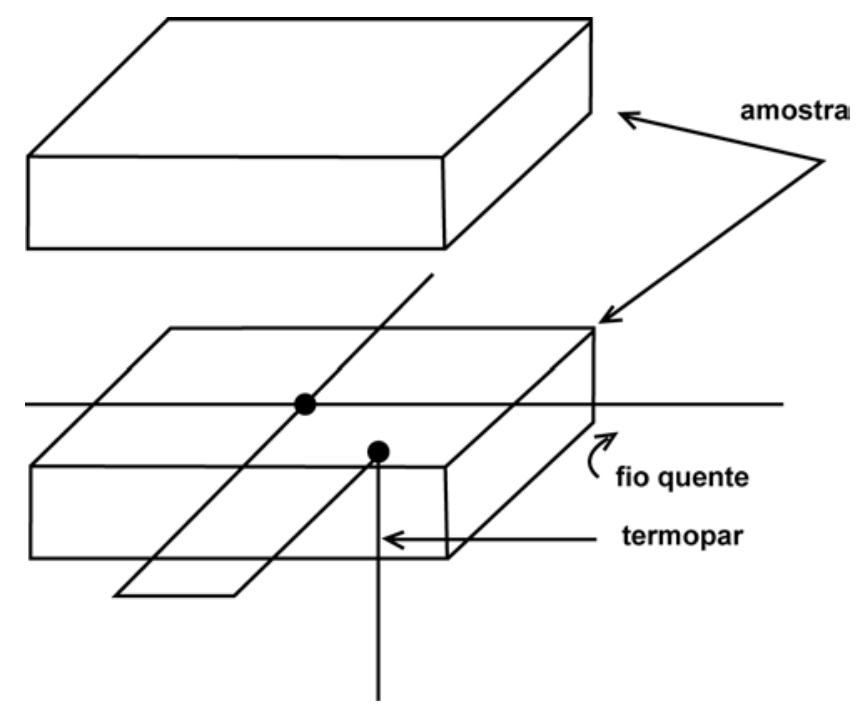

Figura 3: Técnica padrão de fio quente.

[Figure 3: Hot wire standard technique.]

$$
\mathrm{k}=\frac{\mathrm{q}^{\prime}}{4 \pi} \frac{\ln \left(\mathrm{t}_{2} / \mathrm{t}_{1}\right)}{\mathrm{T}_{2}-\mathrm{T}_{1}}
$$

onde: $\mathrm{k}=$ condutividade térmica do material $(\mathrm{W} / \mathrm{mK})$; $\mathrm{q}^{\prime}=$ densidade linear de potência $(\mathrm{W} / \mathrm{m}) ; \mathrm{t}_{1}, \mathrm{t}_{2}=$ tempos contados a partir do início da liberação de calor (s) e $\mathrm{T}_{1}, \mathrm{~T}_{2}$ $=$ excessos de temperatura no fio quente nos tempos $\mathrm{t}_{1}$ e $\mathrm{t}_{2}$, respectivamente, em relação à temperatura de referência $(\mathrm{K})$.

$$
\mathrm{q}^{\prime}=\frac{\mathrm{V}^{2}}{\mathrm{R}} \quad \text { ou } \quad \mathrm{q}^{\prime}=\mathrm{RI}^{2} \quad \text { ou ainda } \quad \mathrm{q}^{\prime}=\mathrm{VI}
$$

onde: $\mathrm{V}=$ tensão nos terminais do fio quente $(\mathrm{V}), \mathrm{R}=$ resistência elétrica do fio $(\Omega / \mathrm{m})$, e I = corrente que passa pelo fio (A).

Técnica de resistência do fio quente-Nessa técnica, descrita por Morrow [7], a variação da resistência do fio quente devido ao aquecimento é utilizada para medir a temperatura. Uma ponte de Kelvin é empregada para a medida de resistência do fio e, por conseqüência, da temperatura. A Fig. 4 mostra um diagrama do arranjo experimental. As resistências $\mathbf{A}$ e a são iguais entre si, o mesmo acontecendo com as resistências $\mathbf{B}$ e $\mathbf{b}$. A razão A:B é, portanto, igual à razão $\mathrm{X}: \mathrm{S}$ quando não há passagem de corrente elétrica pelo galvanômetro, sendo X a resistência desconhecida e $\mathrm{S}$ uma resistência padrão.

Embora uma ponte de Kelvin seja projetada para trabalhar em equilíbrio, nesse caso, os seus desvios em relação ao equilíbrio, em função do tempo, são registrados, obtendo-se, a partir desses resultados, a curva temperatura em função do tempo, que é utilizada para o cálculo da condutividade térmica do material. Nesse método deve ser usada uma resistência elétrica cuja relação resistência-temperatura seja muito bem conhecida. Na prática, um registrador gráfico $\mathrm{x}$-t, ou um computador pode ser utilizado para registrar o desequilíbrio da ponte em função do tempo. A partir desse registro calcula-se a resistência do fio em função do tempo. Resistências elétricas em dois tempos diferentes são convertidas em temperaturas nesses tempos, e com auxilio da equação $(\mathrm{G})$ calcula-se a condutividade térmica do material. O procedimento de colocação do fio quente na amostra, e os cuidados a serem tomados com as amostras são análogos

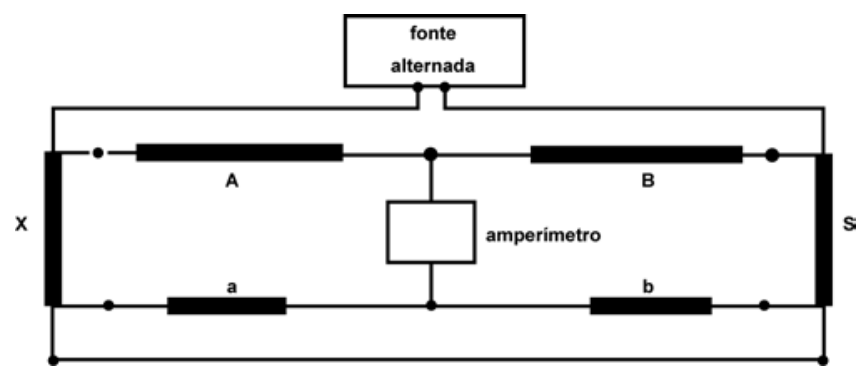

Figura 4: Técnica de resistência de fio quente.

[Figure 4: Hot wire resistance technique.] 
àqueles descritos anteriormente. Qualquer material pode ser usado como fio quente, desde que a relação resistênciatemperatura seja bem conhecida.

Técnica de dois termopares - Para se utilizar essa técnica também são necessários dois corpos de prova. Em um dos corpos de prova são feitos três sulcos paralelos. Pelo sulco central passa o fio quente e pelos outros dois localizados, respectivamente, à distância $\mathrm{r}_{1}$ e $\mathrm{r}_{2}$ do fio, passa o termopar. A Fig. 5 ilustra o arranjo experimental descrito.

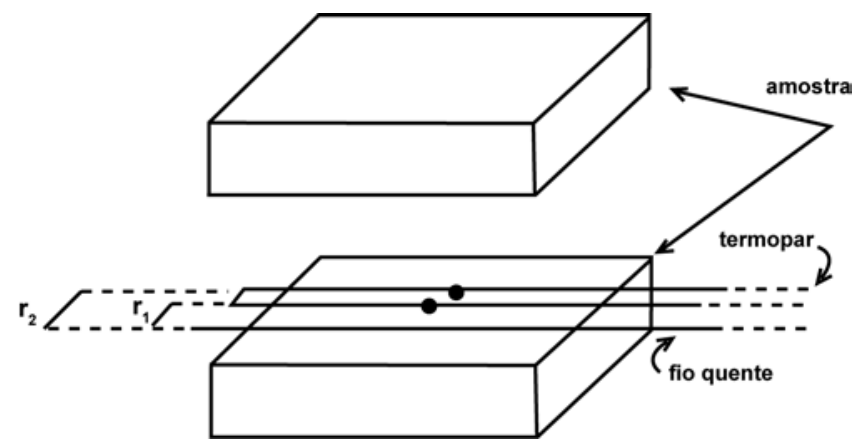

Figura 5: Técnica de dois termopares.

[Figure 5: Two thermocouples technique.]

Nessa técnica faz-se passar uma corrente elétrica constante através do fio, e registra-se a variação da diferença de temperatura entre os pontos à distância $r_{1}$ e $r_{2}$ em função do tempo. A condutividade térmica é calculada de acordo com a equação:

$$
\mathrm{k}=\frac{\mathrm{q}^{\prime}}{2 \pi \mathrm{T}_{\infty}} \ln \frac{\mathrm{r}_{2}}{\mathrm{r}_{1}}
$$

onde: $\mathrm{k}=$ condutividade térmica $(\mathrm{W} / \mathrm{mK}) ; \mathrm{q}^{\prime}=$ densidade linear de potência $(\mathrm{W} / \mathrm{m}) ; \mathrm{r}_{1}, \mathrm{r}_{2}$, = distâncias entre o fio quente e as junções 1 e 2 do termopar $(m)$ respectivamente e $\mathrm{T}_{\infty}=$ diferença de temperatura entre os pontos 1 e $2(\mathrm{~K})$, após ser atingido o estado estacionário.

A temperatura $T_{\infty}$ é obtida a partir do gráfico da temperatura T medida experimentalmente em função da variável $1 / t$, onde $t$ é o tempo de medida, contado a partir do início da passagem da corrente elétrica pelo fio quente. O gráfico que se obtémé uma reta que intercepta o eixo $\mathrm{T}$ das ordenadas no ponto $\mathrm{T}_{\infty}$, quando $1 / \mathrm{t}=$ 0 , o que corresponde a $\mathrm{t} \rightarrow \infty$. O ponto $\mathrm{T}_{\infty}$ corresponde, portanto, ao valor estacionário da diferença de temperatura. A técnica nesse caso é tratada como se fosse um método estacionário.

As técnicas e os cuidados na colocação do fio quente e do termopar são os mesmos já descritos anteriormente. Valores típicos de $r_{1}$ e $r_{2}$ são, respectivamente, 7 e $20 \mathrm{~mm}$.

Técnica de fio quente paralelo - Essa técnica se aplica a amostras homogêneas, porosas ou densas, com densidade superior a $500 \mathrm{~kg} / \mathrm{m}^{3}$, e é possível medir condutividades térmicas até $25 \mathrm{~W} / \mathrm{mK}$ [5]. No caso de materiais densos, em um dos corpos de prova são feitas duas ranhuras paralelas: por uma delas passa o fio quente e pela outra passa um termopar. Novamente, as técnicas e os cuidados na colocação do fio quente e do termopar, e na montagem do arranjo experimental são os mesmos descritos anteriormente. A junção de referência do termopar é mantida a uma temperatura constante (referência), que pode ser gelo fundente, ou então um segundo par de meios tijolos. A Fig. 6 mostra um diagrama do arranjo experimental.

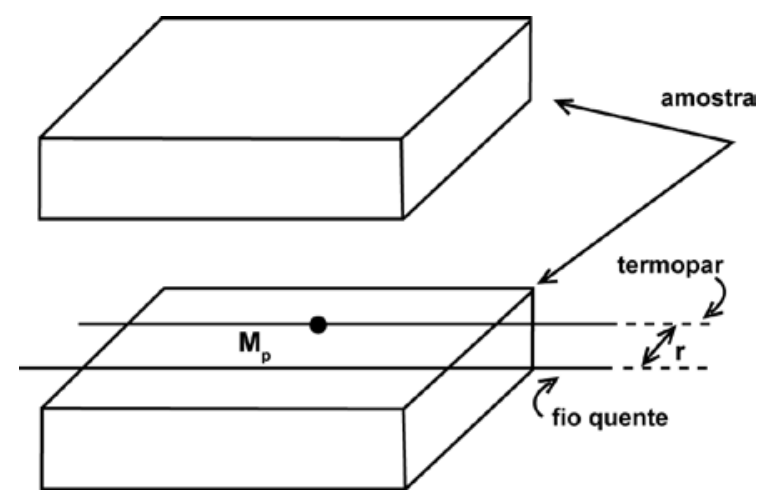

Figura 6: Técnica de fio quente paralelo. [Figure 6: Hot wire parallel technique.]

Para se medir a condutividade térmica faz-se passar uma corrente elétrica constante através do fio, e registra-se o aumento de temperatura no material no ponto $\mathrm{M}_{\mathrm{p}}$ a uma distância $\mathrm{r}$ do fio quente, a partir da temperatura inicial de equilíbrio. No caso de medidas em temperaturas superiores a temperatura ambiente, os corpos de prova e o dispositivo de medida são colocados dentro de um forno. A condutividade térmica é calculada segundo a equação:

$$
k=\frac{-q^{\prime}}{4 \pi T(t)} E_{i}\left(\frac{-\rho c_{p} r^{2}}{4 k t}\right)
$$

onde: $\mathrm{k}=$ condutividade térmica $(\mathrm{W} / \mathrm{mK}), \mathrm{q}^{\prime}=$ densidade linear de potência $(\mathrm{W} / \mathrm{m}), \mathrm{T}=$ excesso de temperatura em relação à temperatura inicial de referência $(\mathrm{K}), \rho=$ densidade do material $\left(\mathrm{kg} / \mathrm{m}^{3}\right), \mathrm{c}_{\mathrm{p}}=$ calor específico à pressão constante $(\mathrm{J} / \mathrm{kgK}), \mathrm{r}=$ distância radial a partir do fio quente $(\mathrm{m}), \mathrm{t}=$ tempo, contado a partir do início da liberação de calor (s) e$\mathrm{Ei}$ = função exponencial integral. A distância $\mathrm{r}$ recomendada entre o fio quente e o termopar é de 15 a $17 \mathrm{~mm}$.

Essa técnica de medida foi normalizada, em 1978, pela norma DIN 51046-Parte 2 [10]. De acordo com essa norma, o cálculo da condutividade térmica é feito utilizando-se apenas dois pontos do transiente térmico. Quando se pretende considerar um trecho do transiente térmico experimental, a condutividade térmica é então calculada de dois em dois pontos e, em seguida, é feita uma simples média aritmética entre os valores calculados. 


\section{EVOLUÇÕES DA TÉCNICA DE FIO QUENTE NO BRASIL}

As primeiras medidas de condutividade térmica no Brasil utilizando a técnica de fio quente foram realizadas em 1984 [11]. A variante utilizada foi a técnica padrão, e o arranjo experimental era bastante simples: uma fonte de tensão, uma resistência de kanthal (fio quente), um termopar ferro-constantam, um voltímetro e um cronômetro. As medidas eram feitas somente à temperatura ambiente. A condutividade térmica era então calculada pela equação $\mathrm{G}$. A Fig. 7 mostra o arranjo experimental utilizado.

Em 1986 foi publicado no Brasil [12] o primeiro trabalho utilizando a técnica de fio quente. Nesse trabalho, que utiliza a variante em paralelo é proposto um novo procedimento de cálculo, substituindo assim a metodologia proposta pela norma DIN 51046-Parte 2. Neste novo procedimento todos

\section{voltímetro}

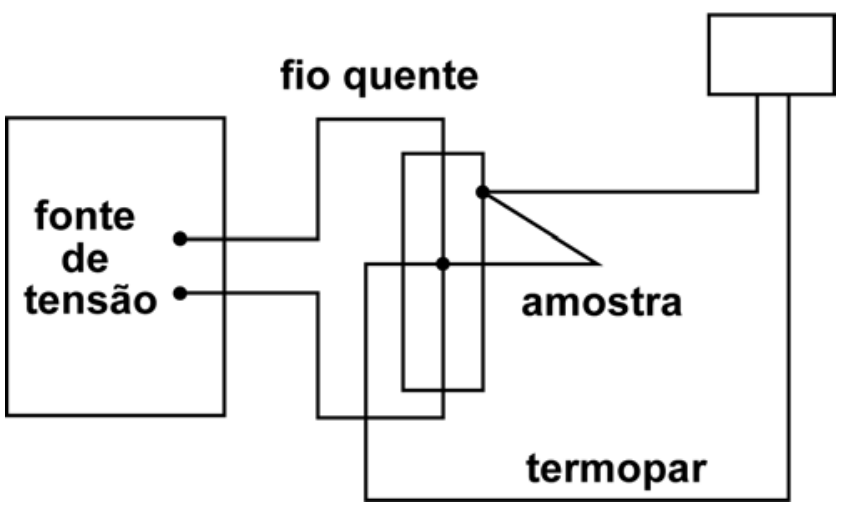

Figura 7: Primeiro arranjo experimental utilizado no Brasil.

[Figure 7: First experimental Brazilian set-up.]

os pontos do transiente térmico registrado experimental são considerados no cálculo. Nesse caso é feito um duplo ajuste por regressão não-linear, obtendo-se simultaneamente, a partir do mesmo transiente térmico experimental, a condutividade térmica, a difusividade térmica e o calor específico. Essa é uma ferramenta matemática muito útil, pois permite a determinação simultânea das três propriedades térmicas num mesmo experimento.

Em 1991, com o objetivo de se comparar numericamente as 4 variantes do método, foi medida a condutividade térmica de dois materiais [6]: um refratário isolante e um sílicoaluminoso. As medidas foram feitas à temperatura ambiente. Os resultados obtidos são mostrados na Tabela I.

Verifica-se pela Tabela I que os resultados obtidos pelo método do fio quente estão bastante próximos, sendo de apenas $6 \%$ a diferença entre o maior e o menor valor. Verifica-se também que o resultado obtido pelo método calorimétrico também está bem próximo dos valores obtidos pelo método do fio quente. Entretanto, é
Tabela I - Comparação de resultados: $\mathrm{k}(\mathrm{W} / \mathrm{mK})$. [Table I-Results and comparisons: $\mathrm{k}(\mathrm{W} / \mathrm{mK})$.]

\begin{tabular}{lccccc}
\hline $\begin{array}{l}\text { Material Técnica } \\
\text { padrão }\end{array}$ & $\begin{array}{c}\text { Técnica } \\
\text { de dois } \\
\text { termopares }\end{array}$ & $\begin{array}{c}\text { Técnica } \\
\text { em } \\
\text { paralelo resistência }\end{array}$ & $\begin{array}{c}\text { Técnica } \\
\text { de }\end{array}$ & $\begin{array}{c}\text { Método } \\
\text { calorimétrico }\end{array}$ \\
\hline Isolante & 0,275 & 0,259 & 0,260 & 0,265 & 0,246 \\
Refratário & 1,278 & 1,251 & 1,265 & - & 1,223 \\
\hline
\end{tabular}

importante ressaltar que esse valor $(0,246 \mathrm{~W} / \mathrm{mK})$, obtido pelo método calorimétrico, não é o resultado de uma medida experimental direta, mas sim, uma extrapolação a partir de medidas realizadas em temperaturas superiores a $400{ }^{\circ} \mathrm{C}$, uma vez que não é possível utilizar esse método para medidas em temperatura ambiente. Essa é, sem dúvida, uma das grandes vantagens do método de fio quente: é possível medir a condutividade térmica e o calor específico de um material, desde a temperatura ambiente até altas temperaturas.

Em 2002 foi proposta [13] uma pequena modificação na forma de detecção do transiente de temperatura, sugerindo, assim, o que seria uma nova variante da técnica de fio quente, e que foi chamada de técnica de fio quente de superfície. A Fig. 8 mostra o arranjo experimental desta técnica.

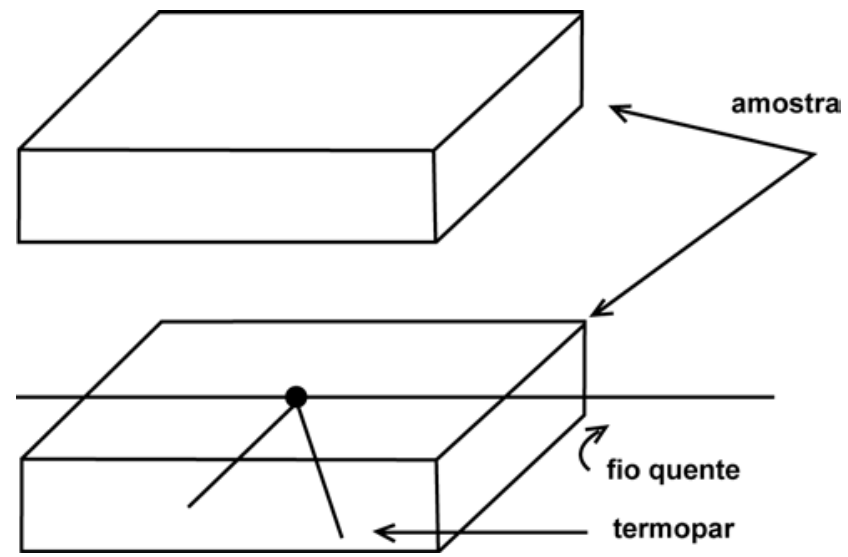

Figura 8: Técnica de fio quente de superfície.

[Figure : Hot wire surface technique.]

Na técnica de fio quente paralelo a condutividade térmica é calculada pela equação I, a qual é rescrita aqui:

$$
k=\frac{-q^{\prime}}{4 \pi T(t)} E_{i}\left(\frac{-\rho c_{p} r^{2}}{4 k t}\right)
$$

onde: $\mathrm{k}=$ condutividade térmica $(\mathrm{W} / \mathrm{mK})$, q' $=$ densidade linear de potência $(\mathrm{W} / \mathrm{m}), \mathrm{T}=$ excesso de temperatura em relação à temperatura inicial de referência $(\mathrm{K}), \rho=$ densidade do material $\left(\mathrm{kg} / \mathrm{m}^{3}\right), \mathrm{c}_{\mathrm{p}}=$ calor específico à pressão constante 
$(\mathrm{J} / \mathrm{kgK}), \mathrm{r}=$ distância radial a partir do fio quente $(\mathrm{m}), \mathrm{t}=$ tempo, contado a partir do início da liberação de calor (s) e $-E i=$ função exponencial integral.

Quando a temperatura é medida na superfície do fio quente (técnica de fio quente de superfície), a equação final para o cálculo da condutividade térmica é obtida a partir das equações $\mathrm{D}$ e $\mathrm{F}$, fazendo-se a seguinte aproximação

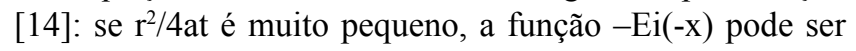
aproximada apenas pelos termos $-\gamma-\ln (x)$. Assim sendo, a temperatura próximo ao fio quente é dada por:

$$
\mathrm{T}=\frac{\mathrm{q}^{\prime}}{4 \pi \mathrm{k}}\left(\ln \frac{4 \mathrm{at}}{\mathrm{r}^{2}}-\gamma\right)
$$

A equação $\mathrm{K}$ mostra que a temperatura próxima ao fio quente é proporcional ao logarítmo do tempo, e a condutividade térmica do meio está contida na constante de proporcionalidade. Isso indica que a condutividade térmica $\mathrm{k}$ pode ser calculada a partir do coeficiente angular da reta temperatura versus logarítmo do tempo. Entretanto, como a fonte de calor (fio quente) não é ideal, e portanto tem uma certa capacidade térmica, e existe uma resistência de contato entre o fio e a amostra, o trecho inicial da curva não é linear, e portanto não deve ser considerado no cálculo da condutividade térmica. A amostra também não é infinita como pressupõe o modelo teórico, e como conseqüência após um certo tempo começa haver perda da linearidade da curva, devido a troca de calor entre a amostra e o meio ambiente, caracterizando também um tempo máximo de medida. Esses tempos mínimo e máximo são determinados experimentalmente a partir do registro da temperatura na superfície do fio quente em função do tempo.

A condutividade térmica é calculada a partir do coeficiente angular da reta temperatura versus tempo, de acordo com a equação $\mathrm{L}$.

$$
\mathrm{k}=\frac{\mathrm{q}^{\prime}}{4 \pi \alpha}
$$

onde q' $=\mathrm{RI}^{2}$ é densidade linear de potência $(\mathrm{W} / \mathrm{m})$, $\mathrm{R}$ é a resistência do fio quente $(\Omega / \mathrm{m})$, I é a corrente elétrica (A), e $\alpha$ é o coeficiente angular da reta.

A Tabela II mostra resultados experimentais obtidos pela técnica de fio quente de superfície e pela técnica de fio quente paralelo para um conjunto de 6 amostras com condutividades térmicas variando de 0,2 a $14 \mathrm{~W} / \mathrm{mK}$. Os tempos mínimos e máximos utilizados nos cálculos, bem como o coeficiente de correlação R e a variação percentual $\delta$ entre as duas técnicas de medida, são também mostrados nessa tabela.

A Tabela II mostra que os resultados obtidos pela técnica de fio quente de superfície, estão em excelente concordância com os resultados obtidos pela técnica de fio quente paralelo, já normalizada.

A norma DIN 51046-Parte 2 prevê que com a técnica em paralelo é possível medir condutividades térmicas até $25 \mathrm{~W} / \mathrm{mK}$. Entretanto, para materiais com condutividade térmica superior a $15 \mathrm{~W} / \mathrm{mK}$, a medida dessa propriedade torna-se problemática em termos do arranjo experimental. A técnica de embutimento do fio quente e do termopar passa a desempenhar um papel importante no valor da condutividade térmica medida experimentalmente [5], e neste caso, o embutimento adequado do fio quente e do termopar é de fundamental importância na determinação de valores confiáveis de condutividade térmica. Entretanto, o fator que limita a técnica de fio quente paralelo, para materiais de alta condutividade é o tempo máximo permissível para o registro do transiente térmico experimental, uma vez que esses dados devem ser registrados antes que comece a haver troca de calor entre a amostra e o meio ambiente. Quanto maior for a condutividade térmica do material, como regra geral maior será também a sua difusividade térmica, e portanto menor será o intervalo de tempo do transiente térmico experimental a ser utilizado no cálculo da condutividade térmica, podendo neste caso haver um comprometimento na confiabilidade do resultado obtido. Já para condutividades térmicas superiores a $10 \mathrm{~W} / \mathrm{mK}$ o intervalo $\left[\mathrm{t}_{\min }, \mathrm{t}_{\max }\right.$ ] a ser considerado nos cálculos torna-se muito pequeno. Para exemplificar o exposto, para uma amostra com condutividade térmica de aproximadamente $13 \mathrm{~W} / \mathrm{mK}$, os tempos mínimo e máximo considerados nos cálculos utilizando a técnica de fio quente paralelo devem ser respectivamente 12 e 81 segundos. Para

Tabela II - Resultados experimentais. [Table II - Experimental results.]

\begin{tabular}{cccccccccc}
\hline & $\begin{array}{c}\text { F.Q.P. } \\
\mathrm{k}(\mathrm{W} / \mathrm{mK})\end{array}$ & $\mathrm{R}$ & $\begin{array}{c}\mathrm{t}_{\min } \\
(\mathrm{s})\end{array}$ & $\begin{array}{c}\mathrm{t}_{\max } \\
(\mathrm{s})\end{array}$ & $\begin{array}{c}\text { F.Q.S. } \\
\mathrm{k}(\mathrm{W} / \mathrm{mK})\end{array}$ & $\mathrm{R}$ & $\begin{array}{c}\mathrm{t}_{\min } \\
(\mathrm{s})\end{array}$ & $\begin{array}{c}\mathrm{t}_{\max } \\
(\mathrm{s})\end{array}$ & $\begin{array}{c}\delta \\
\%\end{array}$ \\
\hline $\mathrm{A} 1$ & 0,2524 & 0,9999 & 42 & 400 & 0,2525 & 0,9997 & 33 & 5100 & 0,04 \\
$\mathrm{~A} 2$ & 1,2782 & 0,9997 & 42 & 338 & 1,2064 & 0,9918 & 125 & 1930 & 5,95 \\
$\mathrm{~A} 3$ & 4,1034 & 0,9996 & 22 & 177 & 3,8263 & 0,9511 & 70 & 850 & 7,24 \\
$\mathrm{~A} 4$ & 4,2704 & 0,9996 & 17 & 140 & 4,5445 & 0,9834 & 15 & 800 & 6,42 \\
A5 & 11,0601 & 0,9990 & 11 & 88 & 10,3842 & 0,9987 & 10 & 400 & 6,51 \\
$\mathrm{~A} 6$ & 13,7431 & 0,9996 & 12 & 81 & 13,4626 & 0,9382 & 10 & 300 & 2,08 \\
\hline
\end{tabular}

F.Q.P. = técnica de fio quente paralelo, F.Q.S. = técnica de fio quente de superficie. 
um material com condutividade térmica de $25 \mathrm{~W} / \mathrm{mK}$ os tempos mínimo e máximo seriam aproximadamente 9 e 54 segundos, para amostras com as dimensões estabelecidas pela norma. Este intervalo de tempo estritamente pequeno limita o número de pontos experimentais a serem utilizados nos cálculos, e pode sem dúvida comprometer a precisão dos resultados. Esta limitação é parcialmente resolvida quando se utiliza a técnica de fio quente de superfície, pois o tempo disponível para o registro do transiente térmico é maior. Para essa mesma amostra, com condutividade térmica de $13 \mathrm{~W} / \mathrm{mK}$, tomada como exemplo, o intervalo de tempo considerado nos cálculos pode ser $[10,300]$ segundos ao invés de $[12,81]$ segundos na técnica de fio quente paralelo. Assim sendo, o intervalo de tempo, bem como o número de pontos experimentais considerados no cálculo da condutividade térmica é bem maior, garantindo assim um resultado mais preciso e confiável.

Esta é, sem dúvida, a grande vantagem da técnica de fio quente de superfície em relação à técnica de fio quente paralelo. O intervalo de tempo considerado no cálculo é bem maior que aquele que seria utilizado na técnica de fio quente paralelo, obtendo-se assim resultados mais precisos e confiáveis. Acredita-se que com a técnica de fio quente paralelo seja difícil a obtenção de resultados precisos e confiáveis para materiais com condutividade térmica superior a $15 \mathrm{~W} / \mathrm{mK}$.

A determinação correta dos tempos mínimos e máximos para o cálculo das propriedades térmicas é crucial e decisiva na técnica de fio quente, principalmente para materiais com alta difusividade térmica. A influência do intervalo de tempo considerado nos cálculos sobre os valores das propriedades térmicas se torna mais crítico quanto maior for a difusividade térmica do material. Assim sendo, com o objetivo de facilitar a correta escolha do intervalo de tempo a ser utilizado nos cálculos, e

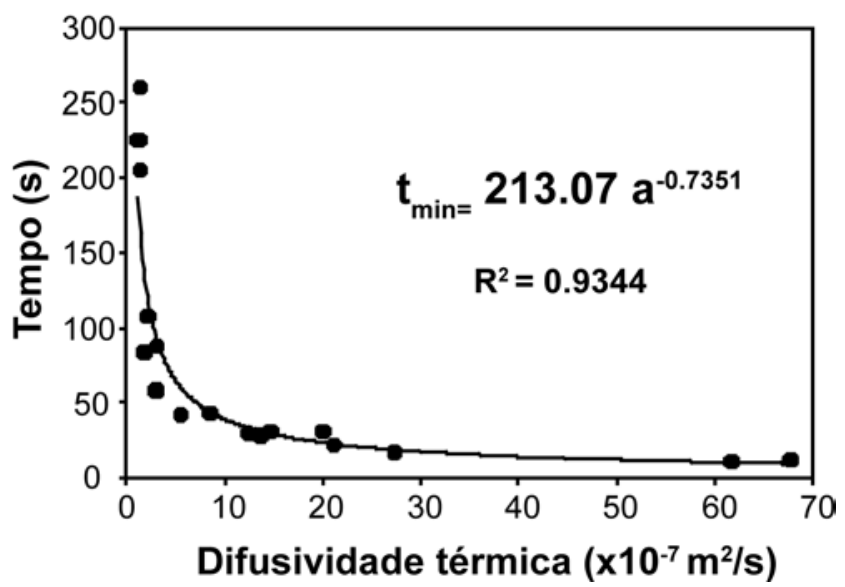

Figura 9: Tempo mínimo de medida em função da difusividade térmica.

[Figure 9: Minimum measuring time as a function of the thermal diffusivity.]

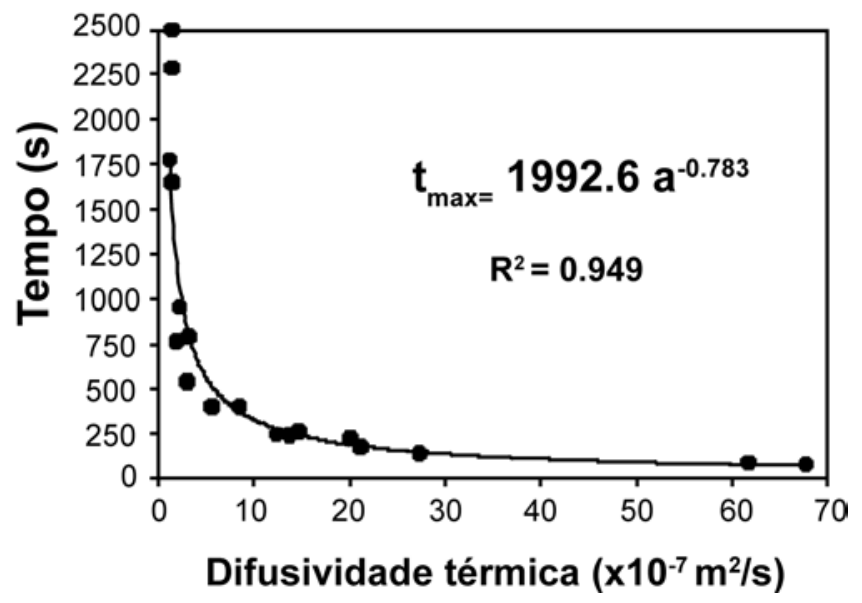

Figura 10: Tempo máximo de medida em função da difusividade térmica.

[Figure 10: Maximum measuring time as a function of the thermal diffusivity.]

utilizando um modelo de simulação numérica, em 2003 foi proposta [15] uma equação empírica relacionando os tempos máximos e mínimos com a difusividade térmica da amostra a ser ensaiada. As Figs. 9 e 10 mostram essas equações.

\section{REPRODUTIBILIDADE}

A reprodutibilidade dos resultados é muito boa desde que os aspectos referentes ao modelo teórico sejam cuidadosamente observados. A Tabela III mostra os desvios percentuais em relação ao valor médio obtido para 5 materiais com condutividade térmica variando desde 0,06 até $10 \mathrm{~W} / \mathrm{mK}$.

Emrelaçãoà condutividade térmica, a reprodutibilidade ainda é muito boa mesmo com um arranjo experimental defeituoso. Todavia desvios do modelo teórico exercem uma influência muito grande no valor do calor específico. Com um arranjo intencionalmente defeituoso no que se refere ao embutimento do fio quente e do termopar foi observado um desvio de apenas $0,5 \%$ na condutividade térmica e $32 \%$ no valor do calor específico, quando comparado com o arranjo experimental correto.

\section{ERRO EXPERIMENTAL}

Em qualquer técnica de medida, a avaliação do erro experimental envolvido é fundamental. Para se fazer uma estimativa do erro experimental associado a esta técnica, podese classificar as principais fontes de erro segundo dois tipos: erros introduzidos pelo procedimento de medida e registro do transiente de temperatura, e erros devido ao procedimento de análise por regressão não linear. No caso da primeira fonte de erros, o erro no resultado final obtido para uma determinada propriedade $P$ pode ser dado por [16]: 
Tabela III - Desvios percentuais em relação ao valor médio.

[Table III - Percent deviations between the average and experimental values.]

\begin{tabular}{|c|c|c|c|c|c|c|}
\hline \multirow[t]{2}{*}{ Amostra } & \multicolumn{2}{|c|}{ Valores Medidos } & \multicolumn{2}{|c|}{ Valor Médio } & \multicolumn{2}{|c|}{$\delta$} \\
\hline & $\begin{array}{c}\mathrm{k} \\
(\mathrm{W} / \mathrm{mK})\end{array}$ & $\begin{array}{c}\mathrm{c} \\
(\mathrm{J} / \mathrm{kgK})\end{array}$ & $\begin{array}{c}\mathrm{k} \\
(\mathrm{W} / \mathrm{mK})\end{array}$ & $\begin{array}{c}\mathrm{c} \\
(\mathrm{J} / \mathrm{kgK})\end{array}$ & $\begin{array}{c}\delta_{\mathrm{k}} \\
(\%)\end{array}$ & $\begin{array}{c}\delta_{\mathrm{c}} \\
(\%)\end{array}$ \\
\hline \multirow{3}{*}{ silicato de cálcio } & 0,0628 & 952,07 & \multirow{3}{*}{0,0631} & \multirow{3}{*}{958,32} & 0,48 & $\overline{0,66}$ \\
\hline & 0,0631 & 954,94 & & & 0,00 & 0,35 \\
\hline & 0,0635 & 967,96 & & & 0,63 & 1,01 \\
\hline \multirow{3}{*}{ vermiculita } & 0,2532 & 613,13 & \multirow{3}{*}{0,2524} & \multirow{3}{*}{613,57} & 0,32 & 0,07 \\
\hline & 0,2512 & 614,05 & & & 0,48 & 0,08 \\
\hline & 0,2528 & 613,54 & & & 0,16 & 0,00 \\
\hline \multirow{3}{*}{ mulita } & 1,9849 & 675,45 & \multirow{3}{*}{1,9932} & \multirow{3}{*}{668,89} & 0,42 & 0,99 \\
\hline & 1,9945 & 660,56 & & & 0,07 & 1,26 \\
\hline & 2,0002 & 670,67 & & & 0,35 & 1,00 \\
\hline aluminoso & 4,2345 & 452,18 & \multirow{3}{*}{4,2704} & \multirow{3}{*}{460,09} & 0,85 & 1,75 \\
\hline \multirow{2}{*}{ (alumina-zirconita) } & 4,2307 & 459,70 & & & 0,94 & 0,00 \\
\hline & 4,3460 & 468,40 & & & 1,77 & 1,81 \\
\hline \multirow{3}{*}{ magnesiano } & 10,2859 & 614,46 & \multirow{3}{*}{10,3589} & \multirow{3}{*}{621,74} & 0,71 & 1,18 \\
\hline & 10,3905 & 606,44 & & & 0,31 & 2,25 \\
\hline & 10,4004 & 644,33 & & & 0,40 & 3,63 \\
\hline
\end{tabular}

$\delta=$ Desvio percentual em relação ao valor médio.

$$
\begin{aligned}
& \delta P=\left[\left(\frac{\partial P}{\partial x_{1}} \delta x_{1}\right)^{2}+\left(\frac{\partial P}{\partial x_{2}} \delta x_{2}\right)^{2}\right. \\
& \left.+\left(\frac{\partial P}{\partial x_{3}} \delta x_{3}\right)^{2}+\cdots+\left(\frac{\partial P}{\partial x_{n}} \delta x_{n}\right)^{2}\right]^{1 / 2}
\end{aligned}
$$

onde $\mathrm{P}=\mathrm{P}\left(\mathrm{x}_{1}, \mathrm{x}_{2}, \mathrm{x}_{3}, \ldots, \mathrm{x}_{\mathrm{n}}\right)$ é um resultado experimental calculado, que é função das variáveis independentes $\mathrm{x}_{1}, \mathrm{x}_{2}, \mathrm{x}_{3}, \ldots$, $\mathrm{x}_{\mathrm{n}} \delta \mathrm{P}$ é o erro associado à propriedade $\mathrm{P}$, e $\delta \mathrm{x}_{1}, \delta \mathrm{x}_{2}, \delta \mathrm{x}_{3}, \ldots, \delta \mathrm{x}_{\mathrm{n}}$ são os erros nas variáveis independentes. Utilizando-se então a equação $M$ verifica-se que o erro percentual introduzido pelo procedimento de registro e medida do transiente de temperatura é da ordem de $\pm 5 \%$. O programa computacional que faz a análise de regressão não linear com ajuste por mínimos quadrados não calcula diretamente o erro no parâmetro ajustado, associado a esse ajuste. Entretanto, para se fazer uma estimativa da qualidade do ajuste é calculado o coeficiente de correlação R em cada uma das medidas experimentais, o qual é dado por [16]: onde $\mathrm{y}_{\mathrm{i}}(\exp )$ são valores experimentais, $\mathrm{y}_{\mathrm{i}}(\mathrm{esp})$ são valores esperados partir do ajuste e $\mathrm{N}$ é o número total de pontos experimentais.

Os coeficientes de correlação calculados normalmente variam entre $\mathrm{R}=0,99$ e $\mathrm{R}=0,99999$. Se o coeficiente de correlação for menor que 0,98 um novo trecho do transiente térmico é selecionado, ou então o processo de medida é repetido. Sabendo-se que a correlação perfeita corresponde a $\mathrm{R}=1$, conclui-se que o erro associado ao procedimento de análise por regressão não linear é muito pequeno, quando comparado com os erros introduzidos pelo procedimento de medida e registro do transiente de temperatura. Assim sendo, o erro total no cálculo das propriedades térmicas é estimado como sendo da ordem de $\pm 5 \%$.

A Fig. 11 mostra o arranjo experimental da técnica de fio quente utilizado no Departamento de Engenharia de Materiais da UFSCar. O sistema de aquisição e processamento de dados é totalmente automatizado: o transiente de temperatura que é detectado pelo termopar é processado em um microcomputador, via um conversor analógico digital.

$$
R=\frac{\sum_{i=1}^{N} y_{i}(\exp ) y_{i}(\operatorname{esp})-\frac{1}{N} \sum_{i=1}^{N} y_{i}(\exp ) \sum_{i=1}^{N} y_{i}(\operatorname{esp})}{\left\{\left[\sum \sum_{i=1}^{N}\left(y_{i}(\exp )\right)^{2}-\frac{1}{N}\left(\sum_{i=1}^{N} y_{i}(\exp )\right)^{2}\right]\left[x\left[\sum_{i=1}^{N}\left(y_{i}(\text { esp })\right)^{2}-\frac{1}{N}\left(\sum_{i=1}^{N} y_{i}(\operatorname{esp})\right)^{2}\right]\right\}^{1 / 2}\right.}
$$




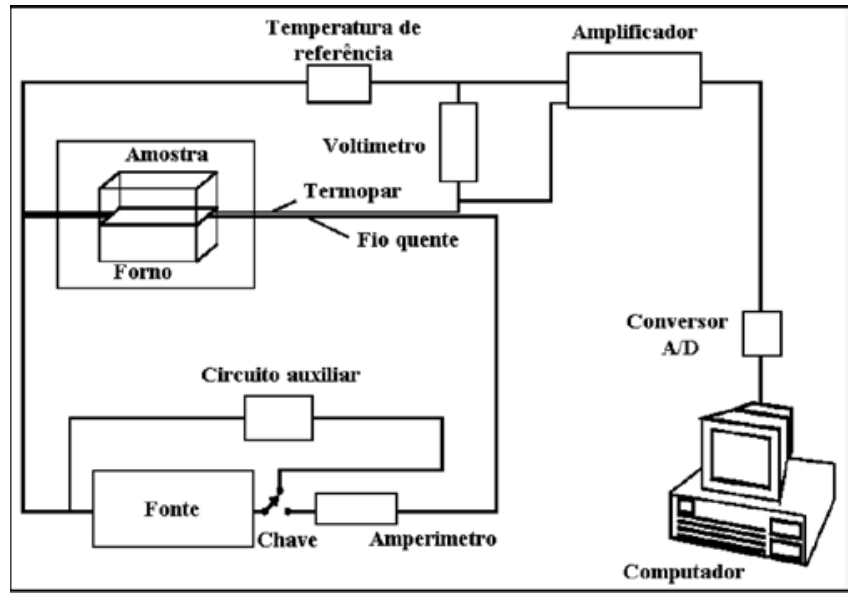

Figura 11: Técnica de fio quente: arranjo experimental-DEMa/ UFSCar.

[Figure 11: Schematic of hot wire equipment-DEMa/UFSCar.]

\section{AGRADECIMENTOS}

O autor agradece ao CNPq (Proc. 302969/2002-0) pelo apoio financeiro.

\section{REFERÊNCIAS}

[1] A. L. Schieirmacher, Wiedemann Ann Phys, 34 (1888) 38.

[2] E. F. M. Van Der Held, F. G. Van Drunen, Physics 15, 10 (1949) 865.

[3] W. E. Haupin, Am. Ceram. Soc. Bull. 39, 3 (1960) 139. [4] H. S. Carslaw, J. C. Jaeger, Conduction of Heat in Solids, Oxford University Press, Oxford (1959) 255.

[5] J. Boer, J. Butter, B. Grosskopf, P. Jeschke, Refractories J. 55 (1980) 22.

[6] W. N. dos Santos, J. S. Cintra Filho, Cerâmica 37, 252 (1991) 101.

[7] G. D. Morrow, Am. Ceram. Soc. Bull. 58, 7 (1979) 687. [8] V. A. Mittenbuhler, Ber. Dtsch. Keram. Ges. 41, 1 (1964) 15.

[9] P. R. E. Staff, Bull. Soc. Fr. Ceram, E1-E2/N3 (1977) 9.

[10] P. R. E. Staff, Bull. Soc. Fr. Ceram, 126, 15 (1980) 15.

[11] W. N. dos Santos, Seminário interno DEMa (1984) 1.

[12] W. N. dos Santos, J. S. Cintra Filho, Cerâmica, 32, 198 (1986) 151.

[13] W. N. dos Santos, Cerâmica 48, 306 (2002) 86.

[14] K. Hayashi, M. Wacamatsu, Proc. Sec. Int. Conf. on Refractories, Kyoto, Japão (1987) 983.

[15] W. N. dos Santos, R. G. Filho, Cerâmica 49, 309 (2003) 29.

[16] J. P. Holman, Experimental Methods for Engineers, McGraw-Hill, New York (1971).

(Rec. 21/02/2005, Ac. 05/08/2005) 